\title{
IDENTIFICATION IN COMPUTER NETWORKS*
}

\author{
Zaw-Sing Su \\ SRI International \\ Menlo Park, California
}

\begin{abstract}
To communicate across a network system, entities within the system need to be able to identify one another. An identifier usually takes the form of a name or an address. Conventions for the assignment of these identifiers and their resolution affect many aspects of network design. Identification is thus a crucial issue for network architecture and standardization. In this paper, our discussion is first devoted to the semantics of names, addresses, and routes, with emphasis on the relationships among them We then examine the implications of layered network architecture, syntactic differences of identifiers, and broadcast communication. Finally, we turn our attention to naming and addressing in large dynamic networks.
\end{abstract}

\section{INTRODUCTION}

To communicate across a network system, entities within a system need to identify one another. An identifier usually takes the form of a name or an address. Conventions for the assignment of these identifiers and their resolution affect many aspects of network design Network interconnection, dynamic reconfiguration and large networks have a profound impact on naming and addressing. Identification has become a crucial issue for network architecture [Oppen81, Clark82, Mills81, Su82] and for network standardization [CCITT82, NBS82]. It has

*This research was supported, in part, by the Naval Flectronic Systems Command under Contract No. No0039-82-C-0399. The views and conclusions contained in this document are those of the author and should not be interpreted as necessarily representing the official policies, either expressed or implied, of the Naval Electronic Systems Command or the U.S. Government.

Permission to copy without fee all or part of this material is granted provided that the copies are not made or distributed for direct commercial advantage, the ACM copyright notice and the title of the publication and its date appear, and notice is given that copying is by permission of the Association for Computing Machinery. To copy otherwise, or to republish, requires a fee and/or specific permission. been the subject of discussion in many recent works [Cerf78, Shoch78, Sunshine82].

Shoch [Shoch78] has given informal definitions of a name, an address, and a route:

The name indicates WHAT we seek, the address WHERE it is, and the route HOW TO GET THERE.

These definitions are useful for differentiating the concepts. However, confusion persists. One of the possible reasons for this confusion appears to be the ambiguity of their semantic relationships. In this paper, we first present the semantics of names, addresses, and routes, with emphasis on the relationships among them To further elaborate, we examine the implications of layered architecture, syntactic differences of identifiers, and broadcast communication. Finally, we turn our attention to naming and addressing in larne dynamic networks.

\section{THE SEMANTICS OF NAMES AND ADDRESSES}

In networking, names are identifiers used for distinguishing entities from one another. In order to distinguish network entities, it is necessary that names be unique. To help identifying a network entity, a narne often incorporates structured information that specifics characterizing attributes of the entity. Such attributes, at the user level for example, may be administrative, geographical, or network topology dependent. However, naming can be accomplished without incorporating any attribute. To illustrate, we borrow the example of U.S. Social Security Number. It is used for resident identification without incorporating characterizing attributes. 
One class of characterizing attributes useful for identification specifies the location of an entity. Thus, we may conclude that addresses are names which incorporate location attributes, but a name may or may not be an address. A corollary to our observation is that an identifier that dœes not incorporate any characterizing attribute can only be a name. When a specific reference is necessary, we will refer to a name that is not an address as addressindependent, and to one that is an address as address-dependent.

For location specification, the characterizing attributes must be defined with respect to a frame of reference, or a "space". Borrowing from mathematical concepts, one may consider location specification to be necessarily functionally related to a metric defined on that space. An address then specifies the location of a "point" in this space. A postal address, for example, specifies a geographic location. One may consider longitude and latitude to be metrics of the geographic address space. Political jurisdiction can be used for postal addressing because it is functionally related to the geographic space. For computer communications, it may not make much sense to address a host by its geographic location, since its geographic location is not necessarily functionally related to the address space for networking. The address space in this case may be defined by the set of network components and the connection relations among them. An address of a host, for example, should specify "where" in the networking system the host is located.

Paralleling Shoch's definitions for a name, an address, and a route, we may further observe that a route characterizes the location of the destination entity by indicating a path for reaching it. Since a route specifies the location it leads to, we may similarly conclude that a route is an address, while an address may or may not be a route. A source route, for example, can be considered an address.

\section{LAYERED NETWORK ARCHITECTURE}

For modularity, a layered approach is often adopted for network architecture [ISO82, Cerf82]. Taking such an approach, the network functions are partitioned into protocd layers. Each layer depends upon lower layer functions and provides services to its upper layers. Naming and addressing are crucial components of each protocol layer. The communicating entities within a layer refer to each other by name. The set of communicating entities at a lower layer may form the basis for the address space of the layer above it. The address of an upper layer entity is often defined as the location of an entity within the lower layer with which the upper layer entity is associated. While an address defines the location of a "point" in the address space, a route can be thought of as defining a "line" leading from the originator to the destination in that space.

An identification space, however, need not necessarily coincide with a protocd layer. It is sometimes found advantageous in network design to introduce an extra identification layer. Such an extra layer may provide additional indirection. An indirection is the flexibility in binding between entities belonging to two different spaces. Logical addressing [Rosen81] is an example of an extra identification layer. It provides the advantages of a fixed-length numeric identifier without completing the binding to a network entity.

\section{SYNTACTIC DIFFERENCES OF IDENTIFIERS}

Specifying conventions for network entity identification is an important aspect of network system design. An identifier may, for example, be in the form of a character or a numeric string of fixed or variable length. In existing computer networks, an identifier of ten takes one of two forms: a variablelength character string or a fixed-length numeric string.

A character string offers user-friendliness. Natural language terms meaningful to human users may be readily incorporated in a character string. Variable-length identification offers the possible accommodation of an arbitrary number of different identifiers. It is also useful for accommodating variation in identification. Communication between entities in two different systems can be accommodated, for example, by employing a common and variablelength addressing scheme. The Xerox internet address is 48 bits long, while the DARPA internet address is 32 bits. Without imposing the identification of one system on the other, one possible scheme to accommodate such a difference, at least in theory, is to employ variable-length addressing concatenating the internet address with a prefix for internet indication. Source routing as a form of addressing [Sunshiner7] is another example of a situation in which variable-length identification comes in handy For a hierarchical identification scheme, a variable-length address may also allow depth variation in the hierarchy. A proposed 
convention for DARPA internet domain naming [Su82] is an example of a hierarchical identification scheme allowing depth variation. The disadvantage of variable-length identification is the inefficiency that results from its excessive processing requirements. However, with the continuing increase in processing speed, variable-length identification may become more and more attractive.

Fixed-length identification imposes a finite limit on the number of different identifiers. This limit is a function of the fixed length and the size of the encoding set (e.g., 16-bit identification provides $2^{16}$. approximately 64,000, different identifiers). Although the limit is usually large enough for practical purposes, it could be significantly reduced when structured information is incorporated. One advantage of fixed-length identifier is that it can be conveniently embedded in a protocol header to allow efficient processing. The 32-bit DARPA internet addressing [Poste181] offers an example of ingenious incorporation of variable-length identification in a fixed- length format.

The difference between a fixed-length numeric string and a variable-length character string is syntactic, while the difference between a name and an address is semantic. The association of names with variable-length character strings and of addresses with fixed-length numerics often leads to the assumption that such an association is required. However, this is not the case. For example "mbxA@hostB.netC", a variable-length character string, can semantically be an address specifying the location of a mailbox "mbxA" on host "hostB" that is connected to network "netC"

Name to address resolution is a binding process. When a name is resolved, it is mapped onto one or more specific addresses. The convention for naming affects when this binding takes place. The later the binding the more flexibility there is for the name-toaddress mapping relation. Address-independent naming offers the flexibility of an indirection. A change of address can be accommodated by accordingly changing the mapping relation without affecting the identities of the communicating entities. Address-dependent naming offers little indirection. The relocation of a communicating entity from one network address to another may imply a change of its identity. However, the use of address-dependent names for identification allows efficient resolution. Resolution for an address-dependent name may involve only a syntactic translation, e.g., a translation from character strings to numeric ones. In the extreme case, when the same identifier is used for both the name and the address, the resolution process can be avoided all together. A proper choice of naming convention depends largely on the networking environment. It can, however, have a significant impact on the system design

\section{BROADCAST COMMUNICATION}

Computer networks initially evolved on the basis of point-to-point communication. This was true at both network and link levels, (e.g. ARPANET). In order to reach a destination in point-to-point communication, the originating entity resolves the destination name into a route, sometimes via an address. The route might be explicitly a source route or implicitly an incremental route. Transmission from an originator proceeds along a single link at a time, propagating toward the destination. Addresses and routes are in particular useful for point-to-point communication.

Broadcasting offers an address-independent mode of communication for computer networking. Broadcast transmission in a local networking environment (e.g., ETHERNET [Metcalfe76]) is an example of broadcast communication at the link level. To broadcast, the originator transmits to all entities attached to the network Often found in practice is a combination of point-to-point communication at a higher level and broadcasting at a lower level. In order to communicate with a specific destination (i.e., point-to-point at the higher level), the originator incorporates a higher level identifier in the transmission specifying the intended destination. The communication is established with the destination recognizing its identity, i.e., its name not its address.

By the nature of broadcast communication, every transmission from each participant in a communicating cornmunity would be received by all others. Without improvernents in the technology, the resulting overhead of broadcasting communication may render its application over a large communicating community impractical. However, its addressindependent mode of operation potentially provides solutions for the accommodation of multi-homing, multi-casting, and network dynamics [Dalal81]. 


\section{LARGE DYNAMIC NETWORKS}

In this discussion, a large network system refers to a network or an internetwork with a large number of network entities. A critical concern for large networks is to locate the destination. Addressdependent naming can be very useful in that an address-dependent name incorporates at least some hint of where the destination is located. The telephone system is an example of a large network employing address-dependent naming. The telephone number a user of the network uses to identify the destination he is trying to reach is an addressdependent name, or an address. By area code and the prefix for local office, it describes where is the destination within the telephone system.

Networks that are capable of changing dynamically impose challenging requirements for network design. A dynamic networking environment may involve network partitioning, network merging, or mobile network entities [Cerf79]. The principle challenge arises from the fact that the location, and thus the address, of a network entity can change dynamically. In the case of a mobile network entity, its physical movement could cause a change of location with respect to a presumably stationary network environment. This environment constitutes the address space. In the case of network partitioning or merging, the address space itself changes. Such change may in turn affect the "location" of certain network entities relative to the network system

In a dynamic networking environment, addressdependent naming may not be desirable. Any change of address would imply a change of identity. For connection-oriented communication, such as communication over virtual circuits, the connection established based upon identities of the source and destination could not be maintained. Using address-independent naming requires name resolution or broadcast communication. However, either approach can lead to inefficiency when a large communicating community is involved. Naming and addressing in large dynamic network systems remains an area for further investigation.

\section{SUMMARY}

In this paper we discussed the semantics of a name, an address, and a route in computer networks and the relationships among them An address may be a name, and a route may be an address, but their respective converses are not necessarily true. A rigid association of names with variable-length character strings and of addresses with numerics can lead to unnecessary confusion. The difference between a name and an address is semantic, not syntactic.

We observe that specification of naming and addressing conventions is a crucial aspect of the design of each protocol layer. Extra identification layers may be introduced for additional indirection. We also observed that broadcasting offers an address-independent mode of communication, which can be useful for the accommodation of some advanced communication requirements. Addressing and routing are in particular useful for point-to-point communication.

Finally, we pointed out that the underlying design issue for dynamic network systems is to accommodate the continual change of addresses. Architecture for large dynamic network systems remains a challenging area for further research

\section{ACKNOWLEDGMENTS}

The author would like to thank Donald L. Nielson for his encouragement and support, Jose J. Garcia for our numerous discussions on the subject of naming and addressing, and Jon B. Postel for his review of an earlier version of this paper.

\section{REFLRENCES}

[CCITT82] CCITT SG VII, "Proposed Draft Recommendation Reference Model of Open Systems Interconnection for CCITT Applications," Special Rapporteur on Layered Models, Melbourne (March 1982). 
[Cerf78] Cerf, Vinton G. and Peter T. Kirstein, "Issues in Packet-Network Interconnection," Proceedings of the IEEE, vol.66, No. 11, (November 1978).

[Cerf79] Cerf, Vinton G., "Internet Addressing and Naming in a Tactical Environment," IEN 110, Information Processing Techniques Office, Defense Advanced Research Projects Agency, Arlington, Virginia (August 1979).

[Cerf82] Cerf, Vinton G., and Edward Cain, "The US DOD Internet Architecture Model," presented at NATO Symposium on Interoperability of Automated Data Systems, SHAPE Technical Centre, The Hague, Netherlands (November 8-12, 1982).

[Clark78] Clark, David, "A Proposal for Addressing and Routing in the Internet," IEN 46, Laboratory of Computer Science, Massachusetts Institute of Technology, Cambridge, Massachusetts (June 1978).

[Dalal81] Dalal, Yogen K. and Robert S. Printis, "48bit Absolute Internet and Ethernet Host Numbers," Proceedings of the 7th Data Communications Symposium, pp.240-245 (October 1981)

[IS082] International Organization for Standardization, "Reference Model of Open Systems Interconnection," ISO/TC97/SC16, Draft International Standard ISO/DIS/7498 (1982).

[Metcalfe76] Metcalfe, Robert M. and David R. Boggs, "ETHERNET: Distributed Packet Switching for Local Computer Networks," Communications of the Association for Computing Machinery, vol.19, No. 7, pp.395-404 (July 1976).

[Mills81] Mills, David L., "Internet Name Domains," RFC 799, COMSAT Laboratory, Washington, D.C. (September 1981).

[NBS82] National Bureau of Standards, "Naming and Addressing in Computer Based Message Systems," Report No. ICST/CBOS-82-4, (Draft) (August 1982).

[Oppen81] Oppen, Derek C. and Yogen K. Dalal, "The Clearinghouse: A Decentralized Agent for Locating Named Objects in a Distributed Environment," Xerox Office Products Division, Systems Development Department, Palo Alto, California (July 1981).
[Postel81] Postel, Jonathan B., "Address Mappings," RFC 796, Information Sciences Institute, University of Southern California, Marina del Rey, California (September 1981).

[Rosen81] Rosen, Eric C., "Logical Addressing," IEN 183, Bolt Beranek and Newman, Cambridge, Massachusetts (May 1981).

[Shoch78] Shoch, John F., "Inter-Network Naming, Addressing, and Routing," Proceedings of 17th IEEE Computer Society International Conference, pp.72-79 (September 1978).

[Su82] Su, Zaw-Sing and Jonathan B. Postel, "Domain Naming Convention for Internet User Applications," RFC 819, Network Information Center, SRI International, Menlo Park, California, (August 1982).

[Sunshine77] Sunshine, Carl A., "Source Routing in Computer Networks," ACM SIGCOMM Computer Communication Review, vol.7, No. 01, pp.29-33 (January 1977).

[Sunshine82] Sunshine, Carl A., "Addressing Problems in Multi-Network Systems," Proceedings of IEEE INFOCOM 82, Las Vegas, Nevada, pp.311-317 (March-April 1982). 